\title{
Direct evidence for singlet-triplet exciton annihilation in $\pi$-conjugated polymers
}

\author{
E. J. W. List, ${ }^{1,2, *}$ U. Scherf, ${ }^{3}$ K. Müllen, ${ }^{4}$ W. Graupner ${ }^{5, \dagger}$ C.-H. Kim, ${ }^{6}$ and J. Shinar ${ }^{2,6}$ \\ ${ }^{1}$ Christian Doppler Laboratory Advanced Functional Materials, Institute of Solid State Physics, Graz University of Technology, \\ A-8010 Graz, Austria \& Institute of Nanostructured Materials and Photonics, A-8160 Weiz, Austria \\ ${ }^{2}$ Ames Laboratory-USDOE, Iowa State University, Ames, Iowa 50011 \\ ${ }^{3}$ Institut für Physikalische und Theoretische Chemie, Universität Potsdam, D-14476 Golm, Germany \\ ${ }^{4}$ Max-Planck-Institut für Polymerforschung, Mainz, Germany \\ ${ }^{5}$ Department of Physics, Virginia Tech, Blacksburg, Virginia 24060 \\ ${ }^{6}$ Department of Physics and Astronomy, Iowa State University, Ames, Iowa 50011
}

(Received 19 September 2002; published 6 December 2002)

\begin{abstract}
A blend of two conjugated polymers with different optical band gaps and different triplet exciton (TE) magnetic resonance features was studied by means of photoinduced absorption (PA) and photoluminescence detected magnetic resonance. From the latter we find that a reduction of TE's on either of the two polymers enhances the radiative singlet exciton (SE) decay on both polymers nearly identically. The PA results rule out other possible mechanisms to yield this signal, except one: a long-range SE-TE annihilation due to a dipoledipole transfer mechanism. Based on this finding, we calculate the transfer radius and quantify the annihilation process for this system and for other conjugated polymers.
\end{abstract}

DOI: 10.1103/PhysRevB.66.235203 PACS number(s): 78.55.Kz, 71.38. - k, 72.80.Le, 78.20.Ls

\section{INTRODUCTION}

The last decade has witnessed the emergence of conjugated polymers as a major class of organic macromolecules ${ }^{1}$ utilized for many possible applications. ${ }^{2,3}$ Among others, polyfluorene (PFO), and the class of poly( $p$-phenylene)-type ladder-polymers (LPPP) ${ }^{4}$ exhibit very distinct emission, 5,6 absorption and photoinduced absorption (PA) features ${ }^{7,8,9,10}$ which have yielded a comprehensive picture of the processes determining the ongoing dynamics of the excited entities, such as singlet excitons (SE's), triplet excitons (TE's), and polarons in conjugated polymers. While it is well established that in organic crystals TE's act as nonradiative quenching centers for SE's, ${ }^{11}$ little is known on the SE-TE interaction in conjugated polymers. The elucidation of this issue is highly desirable since in polymer light emitting devices (PLED's) injected carriers form TE's with a yield of up to $75 \% .^{12}$ Moreover, as a result of the dipole forbidden transition of TE's to the ground state, the lifetime of the TE is extremely long relative to that of the SE's. This results in a high steadystate TE density ${ }^{13,14}$ and hence a high probability for interacting with other species, such as quenching SE's.

This paper provides the first direct evidence for nonradiative quenching of SE's by TE's in conjugated polymers and an estimate of the strength of this interaction. We exclude other possible mechanisms which may be invoked to explain the TE photoluminescence (PL) detected magnetic resonance (PLDMR) at $g \approx 4$, namely TE-TE annihilation to SE's, ${ }^{15}$ also well known to be significant in small $\pi$-conjugated molecules ${ }^{11}$ and ground-state recovery from TE's. ${ }^{16}$ This is done by studying a guest-host polymer blend, which consists of the blueemitting methyl-substituted LPPP (m-LPPP) host and the orangeemitting poly(perylene-co-diethynylbenzene) (PPDB) guest. ${ }^{17}$ The guest was chosen because both its PL and its TE half field resonance at $g \approx 4$ are distinct from those of the host. Hence one can alter the population of the TE spin sublevels of each of the two polymers separately and simultaneously monitor its effect on the decay of SE's in each of the polymers.

\section{EXPERIMENT}

Two types of PLDMR spectra are presented: the $H$-PLDMR, in which changes in the integrated PL $\left(I_{\mathrm{PL}}\right)$ are measured vs the magnetic field $H$, and the $\lambda$-PLDMR, in which the PL emission spectrum is measured at the field $H$ corresponding to the peak of the resonance. The measurements were performed in a He gas-flow cryostat inside an optically accessible $9.35 \mathrm{GHz} x$-band microwave cavity; the incident microwave power was kept below $810 \mathrm{~mW}$. The PL was excited at $351 / 363 \mathrm{~nm}$ and $515 \mathrm{~nm}$ by a Pockels-cell stabilized $\mathrm{Ar}^{+}$laser. The $H$-PLDMR spectra were measured using a Si-photodiode; the laser line was blocked by an appropriate cutoff filter. The change in $I_{\mathrm{PL}}\left(\Delta I_{\mathrm{PL}}\right)$ induced by the microwaves at the field-for-resonance was detected by feeding the photodiode output into a lock-in amplifier referenced to the microwave chopping frequency $\nu_{c}$. The $\lambda$-PLDMR spectra were recorded by replacing the photodiode by a monochromator and a photomultiplier tube. The PA measurements were described elsewhere. ${ }^{7}$ PA and PLDMR data were recorded at $\nu_{c}$ low enough compared to the lifetimes of the involved recombination processes i.e., $\nu_{c} \tau \leqslant 1$, in order to ensure steady-state conditions.

\section{RESULTS AND DISCUSSION}

Figure 1 shows the absorption and emission spectra of pristine m-LPPP and PPDB, discussed in detail previously. ${ }^{17}$ The emission spectrum of m-LPPP and the absorption spectrum of PPDB show a strong overlap so that blending of m-LPPP with $0.05 \mathrm{wt} \%$ PPDB leads to a strong PL contribution of PPDB (see bottom of Fig. 1). Such efficient energy transfer of SE's from m-LPPP to PPDB sites is explained by 


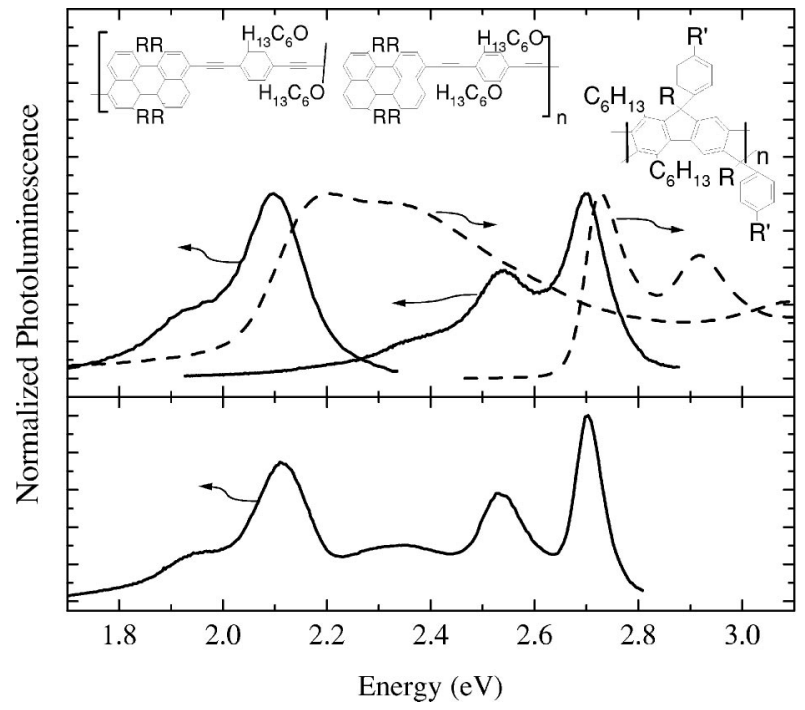

FIG. 1. Top: Chemical structure of (left) PPDB, with $R$ $=$ phenoxy- $t$-butyl, and (right) $\mathrm{m}$-LPPP, $R=\mathrm{CH}_{3} R^{\prime}=\mathrm{C}_{10} \mathrm{H}_{21}$ and $n \approx 6$. Middle: Photoluminescence (full line) and absorption spectrum (dashed line) of PPDB and m-LPPP. Bottom: Photoluminescence spectrum of a blend film of m-LPPP containing $0.05 \mathrm{wt} \%$ PPDB. (300 K).

direct Förster energy transfer ${ }^{24}$ and by excitation energy migration in the m-LPPP matrix and subsequent transfer to PPDB. ${ }^{6}$

Figure 2 shows the half field triplet PLDMR powder pattern of an m-LPPP film, m-LPPP/PPDB blend films of different concentrations, and a pure PPDB film. The inset shows the full-field PLDMR powder pattern of the same films. As clearly observed, an increase of the PPDB content in m-LPPP leads to a more pronounced half field signal at

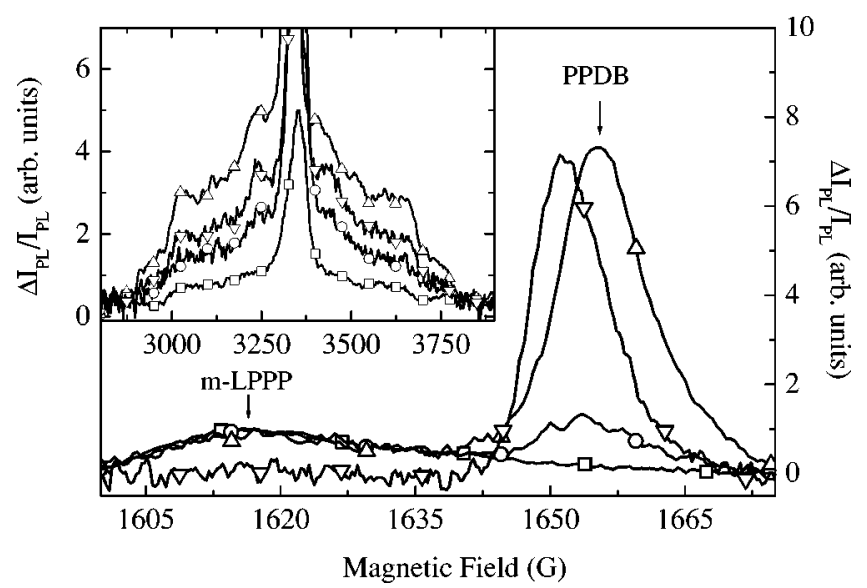

FIG. 2. The half field triplet powder pattern of $\mathrm{m}$-LPPP/PPDB blend films recorded at $20 \mathrm{~K}$ pristine PPDB film (line with up triangles), m-LPPP film with $2.0 \mathrm{wt} \%$ PPDB (line with down triangles), m-LPPP film with 0.05 wt $\%$ PPDB (line with circles) and pristine $\mathrm{m}$-LPPP film (line with squares). The graphs are normalized to the m-LPPP maximum at $1615 \mathrm{G}$. The inset shows the full-field triplet powder spectra of the same films. PL excitation at $\lambda_{\mathrm{EXC}}$ $=515 \mathrm{~nm}$ for PPDB $\lambda_{\mathrm{EXC}}=351 / 363 \mathrm{~nm}$ for all other samples. (The symbols denote the same concentrations as in the half field spectra).

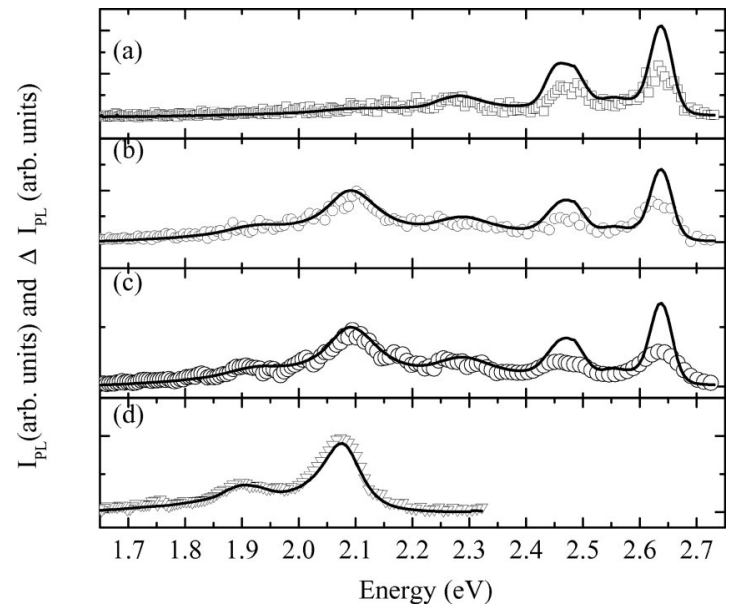

FIG. 3. PL spectra (solid lines) and spectral dependence of the half field triplet PLDMR ( $\lambda$-PLDMR) of $\mathrm{m}$-LPPP/PPDB blend films, recorded at the peak of the resonance (open symbols). (a) Pristine m-LPPP film at $1605 \mathrm{G}$ (b) m-LPPP/0.05 wt \% PPDB at $1605 \mathrm{G}$ and (c) at $1657 \mathrm{G}$, and (d) pristine PPDB film at $1659 \mathrm{G}$. PL excitation at $\chi_{\mathrm{EXC}}=351 / 363 \mathrm{~nm}$ for $a-c$ and $\lambda_{\mathrm{EXC}}=515 \mathrm{~nm}$ for $d$.

$1655 \mathrm{G}$, the resonance position of pristine PPDB TE's. This $g \approx 4$ resonance results from the forbidden $\left|\Delta m_{s}\right|=2$ transitions, which enhance the TE recombination due to the different lifetimes of the triplet $|1\rangle$ and $|-1\rangle$ states, as directly shown for anthracene. ${ }^{18}$ Analysis of the zero-field splitting parameters of m-LPPP and PPDB obtained from the full and half field patterns yields the upper limit of the triplet wave function extent $r_{u b} \approx 4.0 \AA$ for PPDB and $3.4 \AA$ for m-LPPP. ${ }^{7,19}$ This difference in the triplet size is manifest in both the different resonance fields of $\sim 1655$ and $\sim 1615 \mathrm{G}$, respectively, and the different full widths at half maximum of 10 and $25 \mathrm{G}$, respectively.

Figure 3 shows the PL $\left(I_{\mathrm{PL}}\right)$ and $\lambda-\mathrm{PLDMR}\left(\Delta I_{\mathrm{PL}}\right)$ spectra for (a) pristine $\mathrm{m}-\mathrm{LPPP}$ at $\sim 1615 \mathrm{G}$, (b) m-LPPP/0.05 wt $\%$ PPDB blend at $\sim 1615 \mathrm{G}$, (c) the same blend at $\sim 1655$ $\mathrm{G}$, and (d) pristine PPDB at $\sim 1655 \mathrm{G}, \Delta I_{\mathrm{PL}}$ of pristine m-LPPP is weaker relative to $I_{\mathrm{PL}}$ at $E>2.4 \mathrm{eV}$ but it follows the PL spectra at lower energies. For pristine PPDB $\Delta I_{\mathrm{PL}}$ essentially follows the entire PL spectrum. The two $\lambda$-PLDMR spectra of the m-LPPP/PPDB blend, recorded at the peaks of the m-LPPP and PPDB resonances at $\sim 1615$ and $\sim 1655 \mathrm{G}$ respectively, both exhibit the same overall weaker contribution from the high-energy m-LPPP emission at $E>2.4 \mathrm{eV}$ and the more pronounced contribution from the $E<2.3 \mathrm{eV}$ emission due to PPDB. This clearly shows that the TE's on either polymer affect the radiative decay of SE's on both polymers identically.

To explain the striking fact, that the alteration of the TE population on one polymer influences the SE decay rate on an adjacent polymer, we first recall that the $g \approx 4$ resonance was previously attributed to magnetic resonance-enhanced TE-TE annihilation to SE's, which enhances the PL, ${ }^{15}$ and (or) to enhanced repopulation of the ground state which enhances the absorption and thereby the emission. ${ }^{16}$ The latter mechanism is directly ruled out since a repopulation of the PPDB ground state and a corresponding increased ab- 


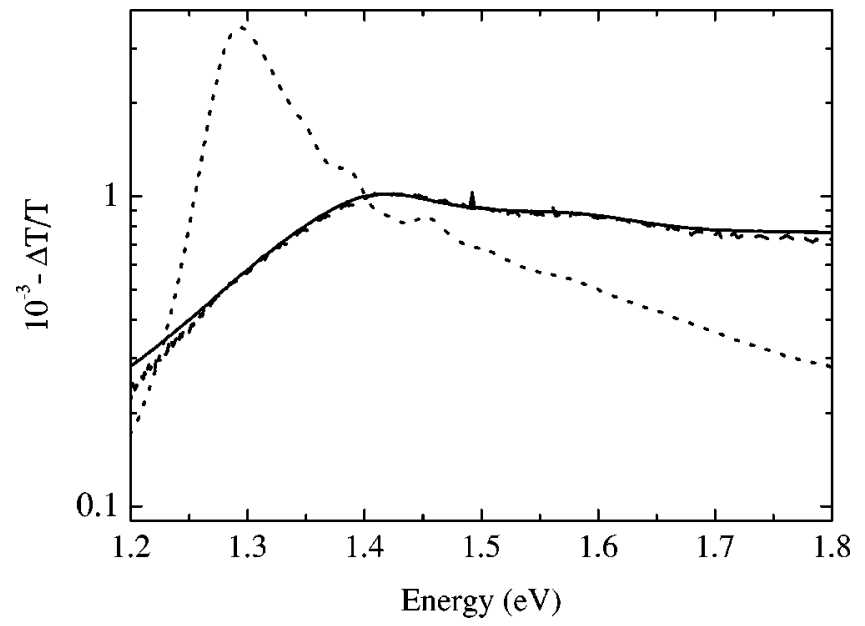

FIG. 4. Photoinduced absorption spectrum of m-LPPP excited at $\lambda_{\mathrm{EXC}}=351 / 363 \mathrm{~nm}$ (dotted line), of pristine PPDB excited at $\lambda_{\mathrm{EXC}}$ $=514 \mathrm{~nm}$ (dashed line), and of an m-LPPP/7.0 wt \% PPDB film excited at $\lambda_{\mathrm{EXC}}=514 \mathrm{~nm}$ (full line). All spectra were recorded at $77 \mathrm{~K}$.

sorption cannot lead to an increased PL from m-LPPP due to the lower optical band gap of PPDB compared to m-LPPP.

The former mechanism can account for the present results only if PPDB TE's are back transferred to m-LPPP TE's to undergo TE-TE annihilation on m-LPPP. Although such a back transfer is unlikely, it was tested by measuring the PA for a rather high-PPDB content of 7 wt \%. Figure 4 shows the PA spectrum of m-LPPP, PPDB, and an m-LPPP/7 wt \% PPDB blend. When excited at an energy larger than the 2.7 eV optical band gap of m-LPPP, the m-LPPP film exhibits a distinct sharp feature at $1.3 \mathrm{eV}$ due to $T_{1} \rightarrow T_{n}$ transitions. ${ }^{9}$ If back-transfer occurs, this PA band should also appear when only PPDB in the m-LPPP/PPDB blend is excited at an energy lower than the optical gap of m-LPPP. Yet despite the high concentration of PPDB molecules in m-LPPP, the PA spectrum of the blend film is identical to that of pure PPDB, ruling out this back transfer process of TE's. We therefore also rule out an enhanced TE-TE annihilation to SE's as the mechanism for the half field triplet PLDMR and assign the resonance to SE-TE annihilation similar to that found in molecular crystals: ${ }^{11}$ At the field-for-resonance at $g \approx 4$ the number of TE's which act as SE quenching centers is reduced, which in turn enhances the PL. We note that the positive spin-1/2 polaron resonance at $g=2$ has been found to be due to a similar polaron-SE annihilation mechanism. ${ }^{20,21,22}$

To explain the long range of the SE-TE annihilation, we invoke Agranovich's theory, ${ }^{23}$ which describes the SE-TE annihilation in molecular crystals by a nonradiative energy transfer, similar to a Förster type transfer, of an excited SE $\left(S_{1}^{*}\right)$ to a TE $\left(T_{1}\right)$ :

$$
T_{1}+S_{1}^{*}=S_{0}+T_{n}+\text { phonons, }
$$

where $S_{0}$ is the singlet ground state and $T_{n}$ an excited state of the TE manifold. ${ }^{24}$ The efficiency of the transfer of the SE energy to photoinduced TE's is determined by the critical radius $R_{\mathrm{SE}-\mathrm{TE}}$ which is the distance at which $50 \%$ of the SE's
TABLE I. Calculated critical radii $R_{\text {SE-TE }}[$ see Eq. (2)], the SE-TE annihilation constant $\gamma_{\mathrm{SE}-\mathrm{TE}}$ and the effective quenching rate constant $k_{\text {SE-TE }}[$ see Eq. (3)] for a SE on a donor molecule and a TE on an acceptor molecule. For the calculations: $D=5$ $\times 10^{-4} \mathrm{~cm}^{2} \mathrm{~s}^{-1}, \quad \eta=0.2, \quad \tau_{\mathrm{SE}} \quad 1 \times 10^{-9} \mathrm{~s}^{-1}, \quad$ and $\quad n_{\mathrm{TE}}=1$ $\times 10^{18} \mathrm{~cm}^{-3}$.

\begin{tabular}{lccc}
\hline \hline $\mathrm{SE}_{\text {Donor }} \rightarrow \mathrm{TE}_{\text {Acceptor }}$ & $R_{\mathrm{SE}-\mathrm{TE}}(\mathrm{nm})$ & $\gamma_{\mathrm{SE}-\mathrm{TE}}\left(\mathrm{cm}^{-3} / \mathrm{s}\right)$ & $k_{\mathrm{SE}-\mathrm{TE}}\left(\mathrm{s}^{-1}\right)$ \\
\hline $\mathrm{SE}_{\mathrm{m}-\mathrm{LPPP}} \rightarrow \mathrm{TE}_{\mathrm{m}-\mathrm{LPPP}}$ & 1.8 & $5.7 \times 10^{-10}$ & $6 \times 10^{8}$ \\
$\mathrm{SE}_{\mathrm{m}-\mathrm{LPPP}} \rightarrow \mathrm{TE}_{\mathrm{PPDB}}$ & 1.9 & $6.2 \times 10^{-10}$ & $6 \times 10^{8}$ \\
$\mathrm{SE}_{\mathrm{PPDB}} \rightarrow \mathrm{TE}_{\mathrm{PPDB}}$ & 3.9 & $1.8 \times 10^{-9}$ & $1 \times 10^{9}$ \\
$\mathrm{SE}_{\mathrm{PPDB}} \rightarrow \mathrm{TE}_{\mathrm{m}-\mathrm{LPPP}}$ & 5.4 & $2.99 \times 10^{-9}$ & $3 \times 10^{9}$ \\
$\mathrm{SE}_{\mathrm{PPV}} \rightarrow \mathrm{TE}_{\mathrm{PPV}}$ & 2.9 & $1.2 \times 10^{-9}$ & $1 \times 10^{9}$ \\
$\mathrm{SE}_{\mathrm{MEH}-\mathrm{PPV}} \rightarrow \mathrm{TE}$ & 3.4 & $1.5 \times 10^{-9}$ & $1 \times 10^{9}$ \\
$\mathrm{SE}_{\mathrm{PFO}} \rightarrow \mathrm{TE}_{\mathrm{PFO}}$ & 1.7 & $5.2 \times 10^{-10}$ & $5 \times 10^{8}$ \\
\hline \hline
\end{tabular}

will be quenched by the TE via Eq. (1). Following the Förster and Agranovich ${ }^{23}$ models one can determine $R_{\mathrm{SE}-\mathrm{TE}}$ from the overlap of the PL spectrum and the $T_{1} \rightarrow T_{n}$ absorption spectrum:

$$
R_{\mathrm{SE}-\mathrm{TE}}^{6}=\frac{9 f^{2} c^{2}}{128 \pi^{3} n_{s}^{4}} \eta_{\mathrm{PL}} \int_{0}^{\infty} \sigma_{\mathrm{TT}}(\nu) f_{\mathrm{SE}}(\nu) \frac{d \nu}{\nu^{4}},
$$

where $c=2.99 \times 10^{8} \mathrm{~m} / \mathrm{s}, f^{2}$ is an orientation factor which is $2 / 3$ for random molecular orientation, $\sigma_{\mathrm{TT}}$ is the triplettriplet absorption cross section, $\eta_{\mathrm{PL}}$ the quantum yield, $n_{s}$ $=1.58$ is the refractive index of the host polymer, and $f_{\mathrm{SE}}(\nu)$ is the normalized PL spectrum $\left[1=\int_{0}^{\infty} f_{\mathrm{SE}}(\nu) d \nu\right]$. To obtain the $T_{1} \rightarrow T_{n}$ absorption spectra the PA spectra were corrected for polaron absorption or were obtained directly from pulse radiolysis measurements. ${ }^{9}$ In addition, $\sigma_{\mathrm{TT}} \sim 4 \times 10^{-16} \mathrm{~cm}^{2}$ was obtained from the molar absorption coefficient as given in Ref. 9, and we assume that the coefficient is similar for all calculations, in good agreement with Ref. 14. The values of $R_{\mathrm{SE}-\mathrm{TE}}$ for several polymers are summarized in Table I.

Comparing the values of $R_{\mathrm{SE}-\mathrm{TE}}$ given in Table I, longrange SE-TE annihilation can well account for the more pronounced influence of the TE's located on PPDB on SE's (larger $\Delta I_{\mathrm{PL}} / I_{\mathrm{PL}}$ ) than that of TE's on m-LPPP on SE's on m-LPPP (see Fig. 3 ). Yet comparing the calculated $R_{\text {SE-TE }}$ with the typical $\sim 0.5 \mathrm{~nm}$ intermolecular distances in conjugated polymers, one finds that SE-TE annihilation also quantitatively explains the observation that an alteration of the TE population on one polymer influences the SE decay rate on an adjacent polymer, both for SE's located on m-LPPP (PPDB) and the TE's on PPDB (m-LPPP). Furthermore the dipole-dipole nature of the SE-TE annihilation mechanism also accounts for the observation that $\Delta I_{\mathrm{PL}}$ of pristine m-LPPP is weaker relative to $I_{\mathrm{PL}}$ at $E>2.4 \mathrm{eV}$. This is a consequence of the weaker relative overlap of $I_{\mathrm{PL}}$ at $E$ $>2.4 \mathrm{eV}$ with the PA absorption at this position. Moreover, the calculated radii given in Table I demonstrate a clear trend: a larger energy difference between emission and TE PA in wide band-gap polymers such as PFO and m-LPPP yields a much weaker SE-TE annihilation probability than in lower band-gap polymers such as poly( $p$-phenylene vinylene) $(\mathrm{PPV}){ }^{25}{\mathrm{MEH}-\mathrm{PPV}^{9} \text { or PPDB. }}^{2}$ 
To quantify the significance of SE-TE annihilation for the $\mathrm{PL}$ or electroluminescence (EL) yield one needs to determine the SE-TE annihilation rate constant $\gamma_{\mathrm{SE}-\mathrm{TE}}$ which, according to Yokota, ${ }^{26}$ is

$$
\gamma_{\mathrm{SE}-\mathrm{TE}}=0.676 \times 4 \pi\left[\frac{\left(R_{\mathrm{SE}-\mathrm{TE}}\right)^{6}}{\tau_{\mathrm{SE}} \eta}\right]^{1 / 4} D^{3 / 4}
$$

with $\tau_{\mathrm{SE}}$ the SE lifetime, $\eta$ the quantum yield of SE's and $D$ the sum of the TE and SE diffusion coefficients $D_{\mathrm{TE}}$ and $D_{\mathrm{SE}}$, respectively. Given the well-established fact that $D_{\mathrm{TE}}$ $\ll D_{\mathrm{SE}},{ }^{11} \quad D \approx D_{\mathrm{SE}}$ which has been experimentally determined. ${ }^{22}$ Typical values of $\gamma_{\mathrm{SE}-\mathrm{TE}}$ are given in Table I. The effective quenching rate constant $k_{\mathrm{SE}-\mathrm{TE}}=\gamma_{\mathrm{SE}-\mathrm{TE}} n_{\mathrm{TE}}$, where $n_{\mathrm{TE}}$ the triplet exciton densities, are given in Table I. For typical $n_{\mathrm{TE}} \sim 10^{18} \mathrm{~cm}^{-3}$ obtained at high excitation densities ${ }^{14} k_{\mathrm{SE}-\mathrm{TE}}$ is found to be of the order of the radiative decay rate of SE's. This is consistent with the observation of nonlinear relaxation processes at higher excitation densities in both polymers and oligomers, ${ }^{27}$ which were previously assigned to the nonlinear SE-SE annihilation mechanism. The present analysis shows that SE-TE annihilation must be considered in future analyses of such nonlinear behavior. Furthermore note that in the EL process, where the yield of TE's can be as high as $75 \%$, i.e., much higher than the $\sim 1 \%$ yield from photoexcitation, ${ }^{14}$ this loss mechanism may be very significant, especially under conditions of electrically pumped lasing, which requires intense carrier injection.

\section{CONCLUSION}

In conclusion, we have unambiguously identified the SE-TE annihilation process in conjugated polymers. We showed that this process is established by a nonradiative dipole-dipole transfer of Förster type and we determined the magnitude of this process for a wide range of conjugated polymers, demonstrating that it is a significant nonradiative decay channel for SE's at high excitation densities.

\section{ACKNOWLEDGMENTS}

We acknowledge the financial support of the FWF-Project No. 12806 and we thank G. Leising and E. Zojer for fruitful discussions. CDL-AFM is a key member of the AT\&S R\&D network. Ames Laboratory is operated by Iowa State University for the U.S. Department of Energy under Contract No. W-7405-Eng-82. The work in Ames was supported by the Director for Energy Research, Office of Basic Energy Sciences.
*Author to whom correspondence should be addressed. Electronic address: e.list@tugraz.at

†Present address: austriamicrosystems AG, Schloss Premstaetten A 8141 Austria/Europe.

${ }^{1}$ C. K. Chiang, C. R. Fincher, Y. W. Park, A. J. Heeger, H. Shirakawa, E. J. Louis, S. C. Gau, and A. G. MacDiarmid, Phys. Rev. Lett. 39, 1098 (1977).

${ }^{2}$ N. S. Sariciftci, L. Smilowitz, A. J. Heeger, and F. Wudl, Science 258, 1474 (1992).

${ }^{3}$ R. H. Friend, R. W. Gymer, A. B. Holmes, J. H. Burroughes, R. N. Marks, C. Taliani, D. D. C. Bradley, D. A. Dos-Santos, J. L. Bredas, M. Logdlund, and W. R. Salaneck, Nature (London) 397, 121 (1999).

${ }^{4}$ U. Scherf and K. Müllen, Makromol. Chem. 12, R489 (1991).

${ }^{5}$ W. Graupner, G. Leising, G. Lanzani, M. Nisoli, S. De Silvestri, and U. Scherf, Phys. Rev. Lett. 76, 847 (1996).

${ }^{6}$ E. J. W. List, C. Creely, G. Leising, N. Schulte, A. D. Schlüter, U. Scherf, K. Müllen, and W. Graupner, Chem. Phys. Lett. 325, 132 (2000).

${ }^{7}$ E. J. W. List, J. Partee, J. Shinar, U. Scherf, K. Müllen, W. Graupner, K. Petritsch, E. Zojer, and G. Leising, Phys. Rev. B 61, 10807 (2000).

${ }^{8}$ D. Hertel, S. Setayesh, H. G. Nothofer, U. Scherf, K. Müllen, and H. Bässler, Adv. Mater. 13, 65 (2001).

${ }^{9}$ A. P. Monkman, H. D. Burrows, M. da G. Miguel, I. Hamblett, and S. Navaratnam, Chem. Phys. Lett. 307, 303 (1999); A. P. Monkman, H. D. Burrows, I. Hamblett, S. Navaratnam, U. Scherf, C. Schmitt, ibid. 327, 111 (2000).

${ }^{10}$ W. Graupner, G. Cerullo, G. Lanzani, M. Nisoli, E. J. W. List, G. Leising, and S. De Silvestri, Phys. Rev. Lett. 81, 3259 (1998); M. Wohlgenannt, W. Graupner, G. Leising, and Z. V. Vardeny, ibid. 82, 3344 (1999).
${ }^{11}$ M. Pope and C. E. Swenberg, Electronic Processes in Organic Crystals, 2nd ed. (Oxford University Press, New York, 1998).

${ }^{12}$ Z. Shuai, D. Beljonne, R. J. Silbey, and J. L. Bredas, Phys. Rev. Lett. 84, 131 (2000); M. Wohlgenannt, K. Tandon, S. Mazumdar, S. Ramasesha, Z. V. Vardeny, Nature (London) 409, 494 (2001).

${ }^{13}$ M. A. Loi, C. Gadermaier, E. J. W. List, G. Leising, W. Graupner, G. Bongiovanni, A. Mura, J.-J. Pireaux, and K. Kaeriyama, Phys. Rev. B 61, 1859 (2000).

${ }^{14}$ A. J. Cadby, P. A. Lane, H. Mellor, S. J. Martin, M. Grell, C. Giebeler, and D. D. C. Bradley, Phys. Rev. B 62, 15604 (2000).

${ }^{15}$ S. Swanson, J. Shinar, A. R. Brown, D. D. C. Bradley, R. H. Friend, P. L. Burn, A. Kraft, and A. B. Holmes, Phys. Rev. B 46, 15072 (1992).

${ }^{16}$ I. Y. Chan, and R. H. Clarke, in Triplet State ODMR Spectroscopy, edited by R. H. Clarke, (Wiley, NY, 1982).

17 Tasch, E. J. W. List, C. Hochfilzer, G. Leising, P. Schlichting, U. Rohr, Y. Geerts, U. Scherf, and K. Müllen, Phys. Rev. B 56, 4479 (1997).

${ }^{18}$ J. U. von Schütz, F. Glückel, W. Steudele, and H. C. Wolf, Chem. Phys. 53, 365 (1980).

${ }^{19}$ E. J. W. List, J. Partee, W. Graupner, J. Shinar, and G. Leising, in Electrical, Optical, and Magnetic Properties of Organic SolidState Materials IV, edited by J. R. Reynolds, A. K.-Y. Jen, L. R. Dalton, M. F. Rubner, and L. Y. Chiang, Mater. Res. Soc. Symp. Proc. No. 488 (Material Research Society, Pittsburgh, 1998), p. 99.

${ }^{20}$ Z. V. Vardeny and X. Wei, Mol. Cryst. Liq. Cryst. 256, 465 (1994).

${ }^{21}$ W. Graupner, J. Partee, J. Shinar, G. Leising, and U. Scherf, Phys. Rev. Lett. 77, 2033 (1996).

${ }^{22}$ E. J. W. List, C.-H. Kim, A. K. Naik, J. Shinar, G. Leising, and W. 
Graupner Phys. Rev. B 64, 155204 (2001).

${ }^{23}$ V. M. Agranovich, Theory of Excitons (Nauka, Moscow, Russia, 1968).

${ }^{24}$ Th. Förster, Ann. Phys. (Leipzig) 2, 55 (1948).

${ }^{25}$ C. Gadermaier, E. J. W. List, P. Markart, W. Graupner, J. Partee, J. Shinar, R. Smith, D. Gin, and G. Leising, Synth. Met. 111, 523 (2000).
${ }^{26}$ M. Yokota and O. Tanimoto, J. Phys. Soc. Jpn. 22, 779 (1967).

${ }^{27}$ G. Kranzelbinder, H. J. Byrne, S. Hallstein, S. Roth, G. Leising, and U. Scherf, Phys. Rev. B 56, 11632 (1997); E. S. Maniloff, V. I. Klimov, and D. W. McBranch, ibid. 56, 1867 (1997); G. J. Denton, N. Tessler, N. T. Harrison, and R. H. Friend, Phys. Rev. Lett. 78, 733 (1997). 